\title{
Treatment Efficacy and Compliance in Patients with Diabetic Macular Edema Treated with Ranibizumab in a Real-Life Setting
}

\author{
Anne-Laurence Best, ${ }^{1}$ Franck Fajnkuchen, ${ }^{1,2}$ Sylvia Nghiem-Buffet $\mathbb{D}^{1},{ }^{1,2}$ Typhaine Grenet, ${ }^{1,2}$ \\ Gabriel Quentel, ${ }^{2}$ Corinne Delahaye-Mazza, ${ }^{2}$ Salomon Y. Cohen, ${ }^{2,3}$ \\ and Audrey Giocanti-Aurégan $\mathbb{D D}^{1}$
}

\author{
${ }^{1}$ Ophthalmology Department, Avicenne Hospital, DHU Vision and Handicaps, APHP, Paris 13 University, Bobigny, France \\ ${ }^{2}$ Centre d'Imagerie et de Laser, Paris, France \\ ${ }^{3}$ Ophthalmology Department, Centre Hospitalier Intercommunal de Créteil, Paris Est University, Créteil, France
}

Correspondence should be addressed to Audrey Giocanti-Aurégan; audreygiocanti@yahoo.fr

Received 22 December 2017; Accepted 18 March 2018; Published 18 April 2018

Academic Editor: Toshinori Murata

Copyright (c) 2018 Anne-Laurence Best et al. This is an open access article distributed under the Creative Commons Attribution License, which permits unrestricted use, distribution, and reproduction in any medium, provided the original work is properly cited.

\begin{abstract}
Purpose. To assess real-life efficacy of ranibizumab and treatment compliance of patients with vision loss secondary to diabetic macular edema (DME). Methods. A retrospective study was conducted in DME patients treated with ranibizumab. Patients were monitored every 4 weeks for visual acuity (VA) and central retinal thickness (CRT) by SD-OCT. All patients received a loading dose of 3 monthly injections followed by retreatments on an as-needed basis. The primary endpoint was the change in VA at M12. Patient compliance to the follow-up and the correlation between the injection number and VA were also investigated. Compliance was compared to that of neovascular age-related macular degeneration (nAMD) patients. Results. Seventy-two eyes of 55 consecutive DME patients were included. At baseline, the mean VA was 56.5 letters and CRT was $470 \mu \mathrm{m}$. At M12, the mean VA was 63.4 letters $(p<0.0001), 31.1 \%$ of patients had a VA $>70$ letters, the mean VA change was +6.9 letters, and the mean CRT was $361.9 \mu \mathrm{m}(p=0.0001)$ after a mean number of 5.33 intravitreal injections. In patients who received $\geq 7$ injections, the VA gain and final VA were significantly higher than in patients who received $<7$ injections. At M12, 25.45\% of DME patients were lost to follow-up versus $16.8 \%$ of nAMD patients $(n=55)$. Discussion/Conclusion. Our study confirms the real-life efficacy of ranibizumab in DME at M12 and the need for a large number of injections to achieve better visual outcomes. We also showed a trend to a lower compliance in diabetic versus nAMD patients.
\end{abstract}

\section{Introduction}

Diabetic macular edema (DME) is the leading cause of decreased vision in diabetic patients with a prevalence of $4.8 \%$ [1]. Its management has improved over the last ten years with the increased availability of therapeutic agents. Laser photocoagulation has long been the reference treatment and has led to a $50 \%$ reduction in visual acuity (VA) decrease at 3 years, but this improvement is not sustained over the long term [2]. Thereafter, intravitreal injections (IVI) of corticosteroids have shown promising results [3-6] but their side effects limit their benefits $[7,8]$. Ranibizumab was the first anti-VEGF agent to show a benefit in terms of VA in the treatment of central DME [9-12] in Phase III studies. In these pivotal studies, the VA gain over the first year varies from +6.8 to +12 letters with a number of IVI ranging between 7 and 12. The visual gain and IVI number depend on the treatment regimen and follow-up strategies used.

The aim of this study was to assess the efficacy and safety of ranibizumab for the treatment of DME in a real-life setting in a French private practice.

\section{Methods}

All consecutive patients with vision loss secondary to DME who received their first IVI of ranibizumab $0.5 \mathrm{mg}$ between June 2012 and June 2015 in a private ophthalmology center 
specialized in retina diseases, CIL (Center for Imaging and Laser) in Paris, were retrospectively included. This study was conducted in accordance with the tenets of the Declaration of Helsinki, and an informed consent was obtained from patients. Approval was obtained from the France Macula Federation ethics committee.

Inclusion criteria were patients $\geq 18$ years old, with type 1 or 2 diabetes with vision loss due to center-involved DME. Both eyes of the same patient could be included.

Exclusion criteria were history of another vitreous or retinal pathology, presence of macular ischemia, stroke or cardiac failure $\leq 3$ months before inclusion, and ocular surgery $\leq 6$ months before inclusion.

For each patient, the systemic data were collected (diabetes type and duration, $\mathrm{HbA1C}$, blood pressure, dyslipidemia, presence of nephropathy, macroangiopathy, sleep apnea syndrome, and type of treatment).

At baseline and during the follow-up, all patients underwent a complete ophthalmologic examination with bestcorrected visual acuity (BCVA) measurement according to the ETDRS scale and slit-lamp and noncontact fundus examination (SuperField Volk). Angiography (Spectralis, Heidelberg Engineering, Heidelberg, Germany) was performed to rule out macular ischemia and to assess the stage of diabetic retinopathy (DR). Spectral-domain optical coherence tomography (SD-OCT) (Spectralis, Heidelberg Engineering, Heidelberg, Germany) was performed to measure the central macular thickness (CRT) and macular volume (MV) during the follow-up. DME was defined by a CRT $\geq 300 \mu \mathrm{m}$.

The treatment regimen followed the 2012 European guidelines for ranibizumab use modified in 2014 [13, 14]. Patients received 3 monthly IVI of ranibizumab during the loading phase, followed by reinjection according to a pro re nata (PRN) regimen. Patients were monitored every 4 weeks with BCVA measurement, fundus examination, and CRT measurement. A decrease in BCVA $>5$ letters and/or a CRT $>300 \mu \mathrm{m}$ were indications for retreatment. In the absence of BCVA improvement after the loading phase, treatment was discontinued. Patients with a VA gain $<5$ letters or a CRT improvement $<10 \%$ from baseline values after 3 IVI were considered as nonresponders.

The primary endpoint was the change in BCVA between baseline and month 12 of follow-up (M12).

Secondary endpoints were the CRT, MV after the loading phase and at M12, number of IVI in the first year of followup, and the assessment of patient compliance. Compliance was assessed through 2 parameters: the prevalence of patients lost to follow-up, that is, patients who stopped their follow-up before the end of the first year, and the prevalence of patients with an irregular follow-up, that is, patients who did not attend the required appointments and missed their examination between M12 and M14, but continued their treatment. The compliance of DME patients was compared to that of a series of consecutive neovascular age-related macular degeneration (nAMD) patients treated with ranibizumab for one year in the same center, during the same period.

2.1. Statistical Analysis. A matched Student parametric test was used for statistical analysis, and a $p$ value $<0.05$ was considered significant. For prevalence comparison, a Fisher's exact test was performed. The statistical analysis was carried out using Prism 7 software.

\section{Results}

Seventy-two eyes of 55 patients treated with ranibizumab injections were included. Seventeen patients $(30.9 \%)$ had bilateral DME, and 38 patients (69.1\%) had unilateral DME. The mean DME duration before the first injection was 20.2 months.

The mean follow-up duration after the first IVI was 19.6 months ( \pm 11.39 months), with a median of 17.87 months. Baseline patient characteristics are presented in Table 1.

Diabetic retinopathy (DR) was mild nonproliferative DR (NPDR) in 5 eyes (7\%), moderate NPDR in 18 eyes (25\%), severe NPDR in 20 eyes (27.8\%), and proliferative DR in 8 eyes $(11.1 \%)$. Laser photocoagulation had been previously performed in 21 eyes $(29.1 \%)$.

Twenty-seven eyes (37.5\%) were not treatment naive: 26 eyes had received macular laser therapy and 1 eye had been treated with IVI of triamcinolone in 2004 prior to inclusion. Forty-five eyes (62.5\%) were treatment naive (Table 2).

3.1. Functional Outcomes. The mean baseline BCVA was $56.5 \pm 11.9$ ETDRS $( \pm$ SD) letters. Five out of the $72(6.9 \%)$ eyes had a baseline BCVA score $>70$ ETDRS (Table 3, Figure 1).

The mean BCVA gain was $+6.4 \pm 7.3$ letters at M3 $(p<$ $0.0001),+6.1 \pm 16.7$ letters at M6 $(p<0.0001),+6.5 \pm 8.5$ letters at M9 $(p<0.0001)$, and $+6.9 \pm 10.2$ ETDRS letters at M12 $(p<0.0001)$. After one year of treatment, 37.8\% (17/45) of patients had a VA gain $\geq 10$ letters and $22.2 \%(10 / 45)$ had $\geq 15$ letters and $31.1 \%(14 / 45)$ had reached the BCVA threshold of $>70$ letters versus only $6.9 \%$ at baseline.

At the end of the first year of follow-up, 2 eyes had lost $\geq 10$ letters.

3.2. Anatomical Outcomes. The mean baseline CRT was $470 \mu \mathrm{m}( \pm 134.5)$. The mean CRT change was $-148 \mu \mathrm{m}$ $( \pm 177)$ at $\mathrm{M} 3$ and $-108.1 \mu \mathrm{m}( \pm 176)$ at M12 (Table 3, Figure 2). CRT was $<300 \mu \mathrm{m}$ in $40 \%(18 / 45)$ of eyes at M12.

The baseline MV was $13.2 \mathrm{~mm}^{3}$. The mean change in MV was $-2 \pm 1.6 \mathrm{~mm}^{3}$ at M3 and $-1.6 \pm 1.6 \mathrm{~mm}^{3}$ at M12 (Table 3, Figure 3).

3.3. Number of Intravitreal Injections. 55 patients (72 eyes) received a mean number of $5.33 \pm 2.1$ injections of ranibizumab over the first year. Nineteen eyes had a follow-up of two years with a mean number of 10.84 IVI.

3.4. Compliance with Treatment. Nine (16.4\%) and 14 (25.45\%) patients (10 and 16 eyes) were lost to follow-up at M6 and M12, respectively. As a result, 41 patients (56 eyes) had at least 12 months of follow-up, but only 33 out of the 55 patients $(60 \%, 45$ eyes) attended the control consultation scheduled between the 12th and 14th month, the others were seen later (i.e., 8 patients-14.5\%-had an irregular follow-up). 
TABLE 1: Baseline characteristics of patients.

\begin{tabular}{lc}
\hline Patient number & $n=55$ \\
Sex & \\
$\quad$ Men & $n=34(61.8 \%)$ \\
$\quad$ Women & $n=21(38.2 \%)$ \\
Type of diabetes & \\
$\quad$ Type 1 & $n=8(15.5 \%)$ \\
$\quad$ Type 2 & $n=47(85.5 \%)$ \\
Age (years), mean $\left( \pm \mathrm{SD}^{*}\right)$ & $66.7( \pm 9.59)$ \\
Duration of diabetes $($ years $)$, mean $( \pm \mathrm{SD})$ & $18.1( \pm 13.29)$ \\
HbA1c, mean $( \pm$ SD) & $7.4 \%( \pm 1.25)$ \\
Insulinotherapy & $n=20(36 \%)$ \\
High blood pressure & $n=34(61.8 \%)$ \\
Dyslipidemia & $n=14(25 \%)$ \\
Nephropathy & $n=15(27 \%)$ \\
Macroangiopathy & $n=2(3.6 \%)$ \\
Sleep apnea syndrome & $n=2(3.6 \%)$ \\
\hline
\end{tabular}

*SD: standard deviation.

TABLE 2: Baseline features of retinopathy, maculopathy, and ophthalmologic history.

\begin{tabular}{lc}
\hline Eye number & $n=72$ \\
NPDR & \\
Mild & $5(7 \%)$ \\
$\quad$ Moderate & $18(25 \%)$ \\
$\quad$ Severe & $20(27.7 \%)$ \\
PDR & $8(11.1 \%)$ \\
Laser photocoagulation & \\
PRP & \\
$\quad$ Ongoing & $22(30.5 \%)$ \\
$\quad$ Completed & $21(29.1 \%)$ \\
Focal/grid & $26(36.1 \%)$ \\
Intravitreal injection history & \\
$\quad$ Corticosteroids & $1(1.3 \%)$ \\
DME duration (months): mean $( \pm$ SD) & $20.2( \pm 25.13)$ \\
Pseudophakic & $18(25 \%)$ \\
Vitreomacular surgery & $4(5.6 \%)$ \\
Epiretinal membrane & $10(13.8 \%)$ \\
High intraocular pressure history & $4(5.5 \%)$ \\
\hline
\end{tabular}

NPRD: nonproliferative diabetic retinopathy; PDR: proliferative diabetic retinopathy; PRP: panretinal photocoagulation; SD: standard deviation; $n$ : number of eyes.

3.5. Baseline Characteristics and Compliance of $n A M D$ Patients. Fifty-five consecutive patients with nAMD seen in the same private practice and requiring ranibizumab IVI since January 2013 and followed over 12 months were also included. We included 41 women and 14 men with a mean age of $85.3( \pm 6.3)$. The mean baseline visual acuity was 61.6 $( \pm 13.5)$ letters.
A mean number of 7.38 consultations were carried out over one year. A mean number of 4.5 IVI were administered over the first year. Only $16.8 \%$ of patients were lost to followup at one year.

\subsection{Subgroup Analysis}

3.6.1. Subanalysis according to the Number of IVI at 1 Year. Two subgroups of patients were defined based on the number of IVI administered during the first year: one group received $<7$ IVI ( $n=30$ eyes) and one received $\geq 7$ IVI ( $n=15$ eyes). Patients who received $<7$ IVT had a baseline BCVA of 55.5 letters and a visual gain of +5.43 letters versus a baseline BCVA of 57.1 letters $(p=0.09)$ and a visual gain of +11.19 letters for patients who received $\geq 7$ IVT. At one year, a mean BCVA of $60.96 \pm 15.66$ letters was achieved in the group that received $<7$ IVT versus $68.26 \pm 6.99$ letters in the group with $\geq 7$ IVT $(p=0.04)$.

3.6.2. Functional Response Subanalysis at 1 Year. Two subgroups were defined according to the functional response after one year of treatment. A subgroup of good responders ( $n=8$ eyes) was defined as a BCVA gain $>15$ letters at 1 year, and a subgroup of poorer responders was defined by a BCVA gain $\leq 15$ letters. The group of poorer responders received fewer IVI than the group of good responders (mean IVI number: 5.59 versus 6.5) over the first year $(p=0.03)$. In the good responder group, the baseline BCVA was 46.9 letters and 58.2 letters in the poorer responders $(p=0.047)$.

3.7. Safety. No case of endophthalmitis was reported during the follow-up. One patient with type 2 diabetes had a stroke 6 weeks after the last IVI. This patient subsequently underwent a complete ophthalmologic evaluation, and the decision was made to discontinue IVI.

\section{Discussion}

The results of our study confirm the efficacy of ranibizumab for the treatment of DME responsible for vision loss in a reallife setting with a VA gain of $+6.9 \pm 10.2$ letters after a mean number of 5.33 IVT over the first year of follow-up.

However, our functional results at 1 year are slightly lower than those reported in pivotal [10] and http://drcr.net studies $[8,12,15]$ which show a gain from +6.5 to +12 letters at M12. This discrepancy could probably be due to an insufficient number of injections in our real-life series. Indeed, in our study, patients received 5.33 IVI with a mean annual number of 7.68 consultations, compared to 7-9.4 IVI in pivotal and DRCR.net studies with a number of consultations generally higher than that of our patients.

In the RISE and RIDE studies [11], patients were injected monthly for 36 months. In this case, the VA gains ranged from +11.9 to +12 letters [16] after one year of follow-up. In Europe, in the RESTORE study [10], with a strict monthly monitoring, the visual gain was +6.8 letters at the end of the first year of treatment with a mean number of 7 IVI. Patients were treated according to a PRN regimen, and the retreatment criterion was strictly functional. 
TABLE 3: Best-corrected visual acuity (BCVA), central retinal thickness (CRT), and macular volume (MV) over the first year of follow-up.

\begin{tabular}{|c|c|c|c|c|c|}
\hline & Baseline & Month 3 & Month 6 & Month 9 & Month 12 \\
\hline Number of eyes & $n=72$ & $n=60$ & $n=58$ & $n=52$ & $n=45$ \\
\hline BCVA (ETDRS letters \pm SD) & $56.5 \pm 11.9$ & $62.9 \pm 12.4$ & $62.6 \pm 13.0$ & $63.0 \pm 12.2$ & $63.4 \pm 13.8$ \\
\hline $\mathrm{CRT}(\mu \mathrm{m} \pm \mathrm{SD})$ & $470 \pm 134.5$ & $322 \pm 97.8$ & $344.7 \pm 122.8$ & $350.5 \pm 99$ & $361.9 \pm 124.8$ \\
\hline $\mathrm{MV}\left(\mathrm{mm}^{3} \pm \mathrm{SD}\right)$ & $13.2 \pm 2.4$ & $11.2 \pm 1.5$ & $11.4 \pm 1.7$ & $11.6 \pm 1.7$ & $11.6 \pm 1.6$ \\
\hline \multirow[t]{2}{*}{ BCVA $>70$ letters } & $5(6.9 \%)$ & $22(36.6 \%)$ & $18(31 \%)$ & $16(30.7 \%)$ & $14(31.1 \%)$ \\
\hline & & $0-3$ months & 0-6 months & 0-9 months & 0-12 months \\
\hline Number of eyes & $n=72$ & $n=60$ & $n=58$ & $n=52$ & $n=45$ \\
\hline BCVA gain (ETDRS letters \pm SD) & & $+6.4 \pm 7.3^{*}$ & $+6.1 \pm 16.7^{*}$ & $+6.5 \pm 8.5^{*}$ & $+6.9 \pm 10.2^{*}$ \\
\hline Change in CRT $(\mu \mathrm{m} \pm \mathrm{SD})$ & & $-148 \pm 177$ & $-125.3 \pm 177$ & $-119.5 \pm 143$ & $-108.1 \pm 176$ \\
\hline Change in $\mathrm{MV}\left(\mathrm{mm}^{3} \pm \mathrm{SD}\right)$ & & $-2 \pm 1.6$ & $-1.8 \pm 1.8$ & $-1.7 \pm 1.4$ & $-1.6 \pm 1.6$ \\
\hline Gain $\geq 10$ letters & & $22(30.5 \%)$ & $19(26.3 \%)$ & $18(25 \%)$ & $17(37.8 \%)$ \\
\hline Gain $\geq 15$ letters & & $9(12.5 \%)$ & $11(15.2 \%)$ & $5(6.9 \%)$ & $10(22.2 \%)$ \\
\hline Loss $\geq 10$ letters & & $1(1.3 \%)$ & $6(8.3 \%)$ & $2(2.7 \%)$ & $3(4.1 \%)$ \\
\hline Loss $\geq 15$ letters & & 0 & $3(4.1 \%)$ & 0 & $2(2.7 \%)$ \\
\hline
\end{tabular}

${ }^{*} p<0.0001$.

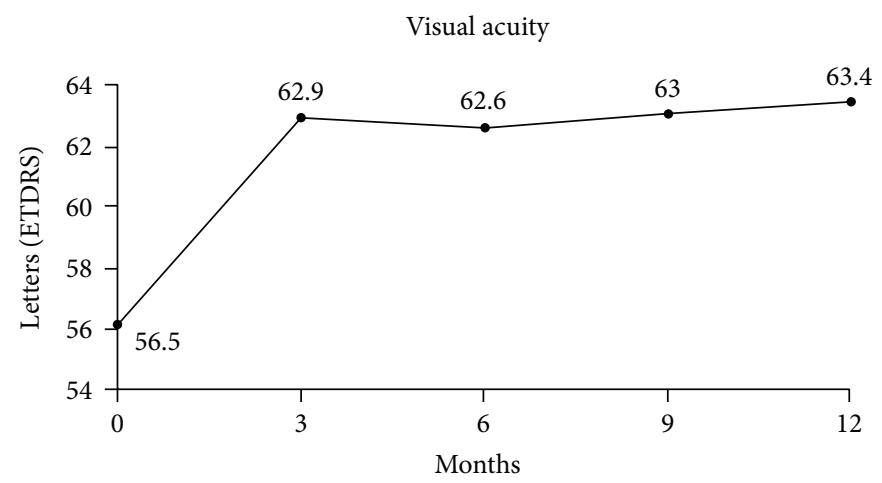

FIGURE 1: Mean change in best-corrected visual acuity over the first year of follow-up.

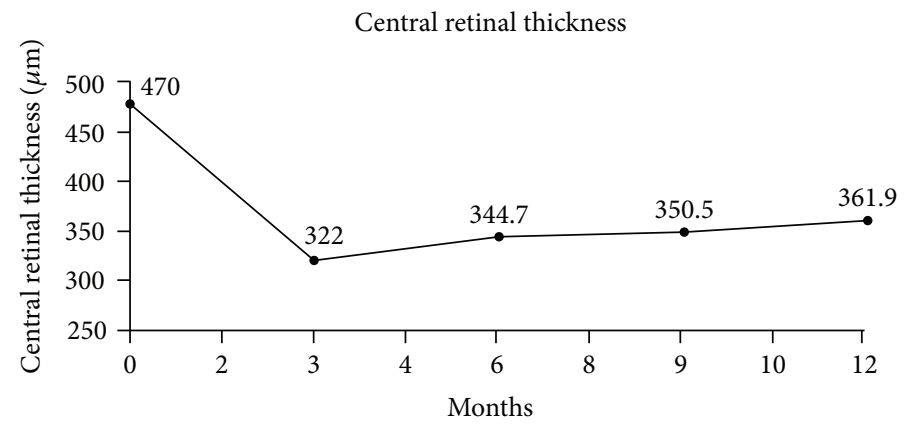

FIGURE 2: Mean change in central retinal thickness over the first year of follow-up.

In the DRCR.net studies [8, 12, 15], ranibizumab IVI were administrated according to a PRN regimen with retreatment based on functional and anatomical outcomes with severe retreatment criteria during the first 6 months to achieve a VA of 20/20 or a dry retina. Thus, patients usually received 5 or 6 injections during the first 24 weeks. With this type of treatment and monitoring every 4 weeks, a gain of +9 letters after 9 IVI was observed with protocol I and +11.2 letters after 10 IVI with protocol T. However, in our study, despite consultations scheduled every 4 weeks, the time between each consultation was longer than 4 weeks in patients who completed the one-year follow-up since they only attended a mean number of 7.68 visits over 12 months.

A clear difference in terms of visual outcomes between the real-life setting and pivotal studies has already been observed in nAMD patients treated with ranibizumab. In 


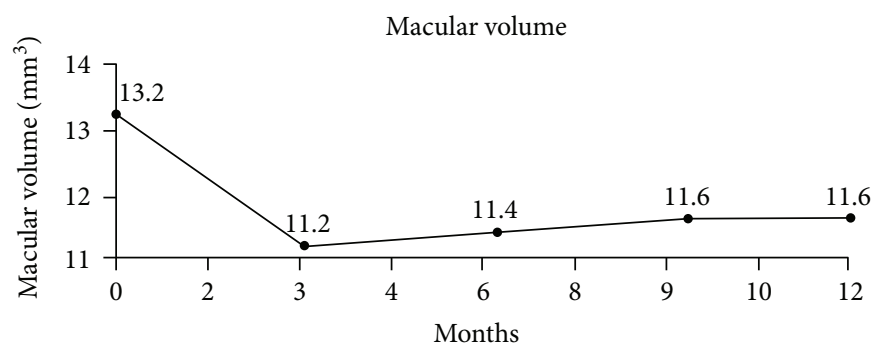

Figure 3: Mean change in macular volume over the first year of follow-up.

nAMD, the MARINA [17] and ANCHOR [18] pivotal studies have shown VA gains ranging between +7.2 and +11.3 letters at one year. The PrONTO study [19] has shown a sustained VA improvement with a personalized PRN regimen and retreatment based on functional and anatomical outcomes allowing a gain of +9.3 letters at one year with twice fewer injections but with a proper monthly follow-up. Real-life studies have shown a smaller improvement with a gain of +4.4 letters at one year for the LUMINOUS [20] study. Another real-life study conducted in our center has shown an even lower visual gain of +0.7 letter after 3.79 IVI and 8.06 consultations over the first year under a PRN regimen, and the authors have concluded on the need for a more regular follow-up with a strict 4-week interval between each consultation. These real-life studies have stressed that there could be a difference in terms of functional outcomes between data from randomized studies with a strict monitoring and treatment protocols and the real-life conditions.

In DME, differences in functional outcomes seem less significant than in nAMD between pivotal and real-life results. The ADMOR real-life study [21] has investigated the efficacy of ranibizumab in patients with DME in South Asia. The results showed a gain of +8.5 letters at 1 year with a mean number of $7 \pm 2$ IVI over the first year. In this study, patients were not strictly monitored every 4 weeks and attended a mean number of $10 \pm 2$ visits during their follow-up. Patients in the ADMOR study had a more severe DME, with an initial VA less than ours (55.3 \pm 13.4 letters), and a higher baseline CRT $(532 \pm 129 \mu \mathrm{m})$. Another reallife study by Hrarat et al. [22] has reported a gain of +10.7 \pm 16.9 letters after 12 months of treatment with a mean number of $5.4 \pm 1.9$ IVI and $8.8 \pm 2.5$ visits during the follow-up. The mean baseline VA was $48.3 \pm 17$ letters, and the baseline CRT was $519.7 \pm 157.3 \mu \mathrm{m}$. This very low baseline VA could explain their high VA gain [16]. A Swedish real-life study by Granström et al. [23] assessing the efficacy of a 12-month treatment with ranibizumab in DME, retrospectively conducted in two ophthalmic departments using a PRN regimen, has reported a gain of +5.2 letters after 12 months of treatment, but the mean number of injections was not specified. Patients had an initial VA greater than ours $(65.0 \pm 12.1$ letters) with a lower initial CRT: $403 \pm 122 \mu \mathrm{m}$.

In our study, with a stricter follow-up and treatment regimen, the VA gains could have probably been greater. This finding is reinforced by a statistically significant correlation between the VA gain and the number of IVI in our study. Patients with more than 7 IVI had a higher VA gain than those who received less than 7 IVI $(p<0.04)$. In addition, the number of injections was greater in the group of patients who had a gain greater than 15 letters compared to the group that did not exceed this threshold $(p<0.03)$.

These results encourage us to adopt a strict follow-up and highlight the need for a regular follow-up by providing appropriate information to patients. Appropriate information is indeed important as the compliance of diabetic patients may be low. Thus, in our series, it should be noted that a significant number of patients were lost to follow-up (25.45\% of patients), suggesting that some diabetic patients are poorly compliant. The small percentage of patients (60\%) who attended the 12 -month consultation supports this hypothesis. This discrepancy between real-life and pivotal studies stresses that real-life studies are necessary to assess the true efficacy of a treatment and to understand the factors limiting efficacy.

The treatment regimen of DME represents a real burden for patients and their family, and diabetic patients must also attend different medical consultations with several specialists and this may be a barrier to a monthly follow-up. Thus, this burden of consultations not only with ophthalmologists could contribute to the lower compliance of diabetic patients compared to that of nAMD patients. Indeed, we assessed in the same private practice 55 consecutive patients with nAMD requiring ranibizumab IVI and followed them over 12 months. They attended a mean number of 7.38 consultations and received a mean number of 4.5 IVI over the first year. Only $16.8 \%$ of patients were lost to follow-up at one year versus $25.45 \%$ in our series of diabetic patients $(p=0.6)$.

Different assumptions may be made regarding the lower compliance of diabetic patients compared to AMD patients: the fact that (i) DME is part of a chronic extraophthalmological disease, diabetes, which, because of its chronicity, may lead to a lassitude with regard to the disease; (ii) the loss of vision is progressive in DME compared to the sudden and often deeper vision loss in nAMD; (iii) diabetic patients are younger and often in the working age, making them less available than nAMD patients who are often retired; and (iv) the cost of the treatment, which may also be a barrier, in particular in a private center where patients must advance the cost. Other studies are needed to confirm the lower compliance of DME patients compared to nAMD patients.

Based on our findings and the results of the literature [24], it seems essential to adopt the treatment regimen to specificities of the diabetic population and to patient availability and preferences after information and, in the case of 
patients who cannot follow a strict monthly regimen to choose the appropriate treatment, for instance, a treat-andextend regimen, providing the same visual outcomes with a lower number of consultations [24] and thus, even despite a possible overtreatment for a few patients.

In conclusion, our real-life study shows a VA improvement in patients with DME, with however a slightly lower gain than that found in pivotal studies after a lower number of IVI. This discrepancy between results obtained in a reallife setting and pivotal studies is not as important as in nAMD despite a higher compliance of nAMD patients in a real-life setting.

This study also shows that the visual outcomes correlate with the number of IVI, and that a strict monthly follow-up is challenging in the real life.

\section{Disclosure}

This study was presented as a paper at the French Society of Ophthalmology in Paris by May 2016 and as a poster at ARVO Meeting in Seattle by May 2016.

\section{Conflicts of Interest}

Dr. Audrey Giocanti-Aurégan reports personal fees from Allergan, Alimera, Bayer, Novartis, and Optos plc outside the submitted work. Dr. Franck Fajnkuchen and Dr. Typhaine Grenet report personal fees from Allergan, Bayer, and Novartis outside the submitted work. Dr. Sylvia Nghiem-Buffet reports personal fees from Allergan, Bayer, Novartis, and Zeiss outside the submitted work. Dr. Corinne Delahaye-Mazza and Dr. Anne-Laurence Best have nothing to disclose. Dr. Gabriel Quentel reports personal fees from Novartis outside the submitted work. Professor Salomon Y. Cohen reports personal fees from Novartis, Bayer, Allergan, Alcon, and Thea outside the submitted work.

\section{Acknowledgments}

This work was supported by an unrestricted grant from AVOPH (Bobigny, France), an association for research and education, and CIL-ASSOC (association for research-Centre d'Imagerie et de Laser, Paris, France).

\section{References}

[1] C. Delcourt, P. Massin, and M. Rosilio, "Epidemiology of diabetic retinopathy: expected $v s$ reported prevalence of cases in the French population," Diabetes \& Metabolism, vol. 35, no. 6, pp. 431-438, 2009.

[2] "Photocoagulation for diabetic macular edema. Early Treatment Diabetic Retinopathy Study report number 1," Archives of Ophthalmology, vol. 103, no. 12, pp. 1796-1806, 1985.

[3] J. A. Haller, B. D. Kuppermann, M. S. Blumenkranz et al., "Randomized controlled trial of an intravitreous dexamethasone drug delivery system in patients with diabetic macular edema," Archives of Ophthalmology, vol. 128, no. 3, pp. 289296, 2010.

[4] Diabetic Retinopathy Clinical Research Network, "A randomized trial comparing intravitreal triamcinolone acetonide and focal/grid photocoagulation for diabetic macular edema," Ophthalmology, vol. 115, no. 9, pp. 1447-1459.e10, 2008.

[5] M. C. Gillies, F. K. P. Sutter, J. M. Simpson, J. Larsson, H. Ali, and M. Zhu, "Intravitreal triamcinolone for refractory diabetic macular edema: two-year results of a double-masked, placebocontrolled, randomized clinical trial," Ophthalmology, vol. 113, no. 9, pp. 1533-1538, 2006.

[6] M. C. Gillies, I. L. McAllister, M. Zhu et al., "Intravitreal triamcinolone prior to laser treatment of diabetic macular edema: 24-month results of a randomized controlled trial," Ophthalmology, vol. 118, no. 5, pp. 866-872, 2011.

[7] M. C. Gillies, J. M. Simpson, C. Gaston et al., "Five-year results of a randomized trial with open-label extension of triamcinolone acetonide for refractory diabetic macular edema," Ophthalmology, vol. 116, no. 11, pp. 2182-2187, 2009.

[8] M. C. Gillies, L. L. Lim, A. Campain et al., "A randomized clinical trial of intravitreal bevacizumab versus intravitreal dexamethasone for diabetic macular edema: the BEVORDEX study," Ophthalmology, vol. 121, no. 12, pp. 2473-2481, 2014.

[9] P. Massin, F. Bandello, J. G. Garweg et al., "Safety and efficacy of ranibizumab in diabetic macular edema (RESOLVE study): a 12-month, randomized, controlled, double-masked, multicenter phase II study," Diabetes Care, vol. 33, no. 11, pp. 2399-2405, 2010.

[10] P. Mitchell, P. Massin, S. Bressler et al., "Three-year patientreported visual function outcomes in diabetic macular edema managed with ranibizumab: the RESTORE extension study," Current Medical Research and Opinion, vol. 31, no. 11, pp. 1967-1975, 2015.

[11] Q. D. Nguyen, D. M. Brown, D. M. Marcus et al., "Ranibizumab for diabetic macular edema: results from 2 phase III randomized trials: RISE and RIDE," Ophthalmology, vol. 119, no. 4, pp. 789-801, 2012.

[12] M. J. Elman, A. Ayala, N. M. Bressler et al., "Intravitreal ranibizumab for diabetic macular edema with prompt versus deferred laser treatment: 5-year randomized trial results," Ophthalmology, vol. 122, no. 2, pp. 375-381, 2015.

[13] Haute Autorité de Santé, "Commission de la transparence Avis," Janvier 2015, https://www.has-sante.fr/portail/upload/ docs/evamed/CT-14433_LUCENTIS_PIS_ETUDE_LUEUR_ Avis1_CT14433.pdf.

[14] F. Bandello, J. Cunha-Vaz, N. V. Chong et al., "New approaches for the treatment of diabetic macular oedema: recommendations by an expert panel," Eye, vol. 26, no. 4, pp. 485-493, 2012.

[15] Diabetic Retinopathy Clinical Research Network, Writing Committee, M. J. Elman et al., "Expanded 2-year follow-up of ranibizumab plus prompt or deferred laser or triamcinolone plus prompt laser for diabetic macular edema," Ophthalmology, vol. 118, no. 4, pp. 609-614, 2011.

[16] P. U. Dugel, J. Hillenkamp, S. Sivaprasad et al., "Baseline visual acuity strongly predicts visual acuity gain in patients with diabetic macular edema following anti-vascular endothelial growth factor treatment across trials," Clinical Ophthalmology, vol. 10, pp. 1103-1110, 2016.

[17] P. J. Rosenfeld, D. M. Brown, J. S. Heier et al., "Ranibizumab for neovascular age-related macular degeneration," The New England Journal of Medicine, vol. 355, no. 14, pp. 1419-1431, 2006.

[18] D. M. Brown, M. Michels, P. K. Kaiser, J. S. Heier, J. P. Sy, and T. Ianchulev, "Ranibizumab versus verteporfin photodynamic 
therapy for neovascular age-related macular degeneration: two-year results of the ANCHOR study," Ophthalmology, vol. 116, no. 1, pp. 57-65.e5, 2009.

[19] G. A. Lalwani, P. J. Rosenfeld, A. E. Fung et al., "A variabledosing regimen with intravitreal ranibizumab for neovascular age-related macular degeneration: year 2 of the PrONTO Study," American Journal of Ophthalmology, vol. 148, no. 1, pp. 43-58.e1, 2009.

[20] F. G. Holz, F. Bandello, M. Gillies et al., "Safety of ranibizumab in routine clinical practice: 1-year retrospective pooled analysis of four European neovascular AMD registries within the LUMINOUS programme," British Journal of Ophthalmology, vol. 97, no. 9, pp. 1161-1167, 2013.

[21] F. Ghanchi and C. A. Hazel, "South Asian diabetic macular oedema treated with ranibizumab (ADMOR) - real-life experience," Eye, vol. 30, no. 1, pp. 133-138, 2016.

[22] L. Hrarat, F. Fajnkuchen, M. Boubaya et al., "Outcomes after a 1-year treatment with ranibizumab for diabetic macular edema in a clinical setting," Ophthalmologica, vol. 236, no. 4, pp. 207-214, 2016.

[23] T. Granström, H. Forsman, A. Lindholm Olinder et al., "Patient-reported outcomes and visual acuity after 12 months of anti-VEGF-treatment for sight-threatening diabetic macular edema in a real world setting," Diabetes Research and Clinical Practice, vol. 121, pp. 157-165, 2016.

[24] C. Prünte, F. Fajnkuchen, S. Mahmood et al., "Ranibizumab $0.5 \mathrm{mg}$ treat-and-extend regimen for diabetic macular oedema: the RETAIN study," British Journal of Ophthalmology, vol. 100, no. 6, pp. 787-795, 2016. 


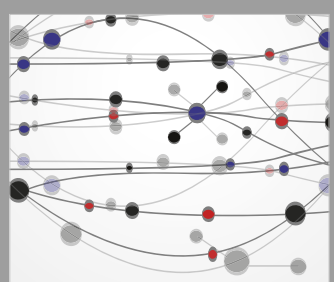

The Scientific World Journal
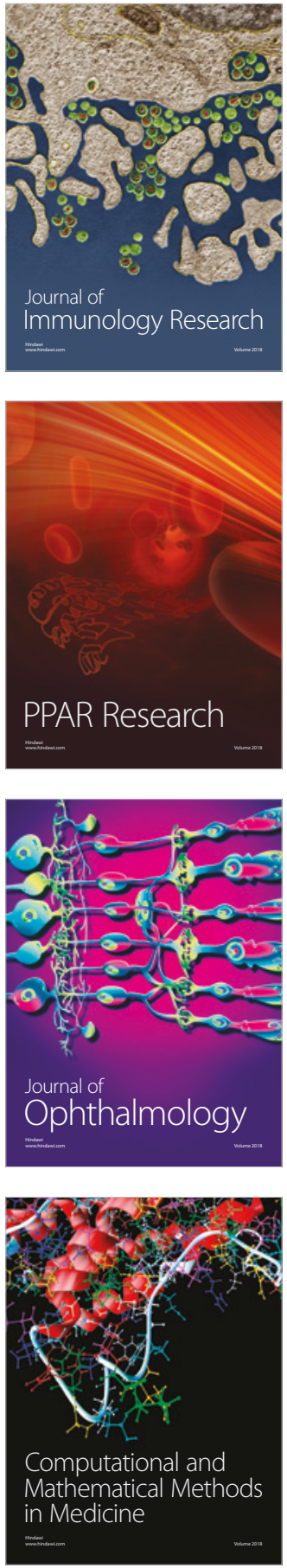

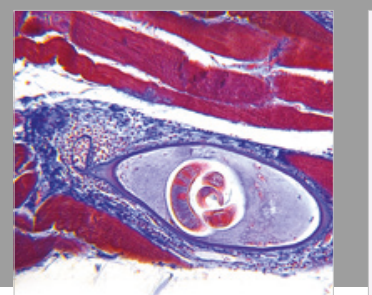

Gastroenterology Research and Practice

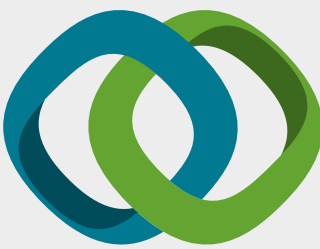

\section{Hindawi}

Submit your manuscripts at

www.hindawi.com
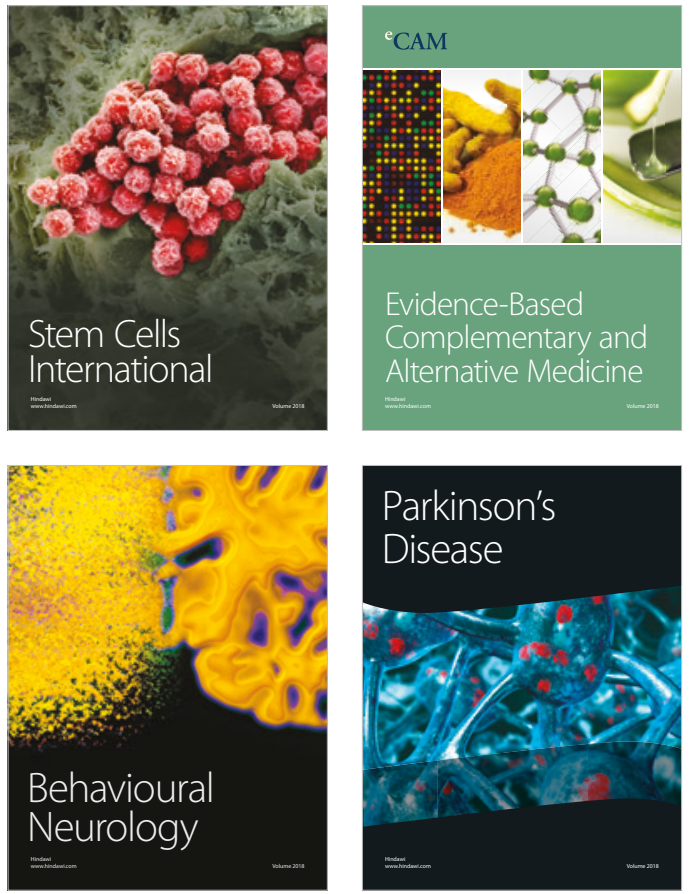

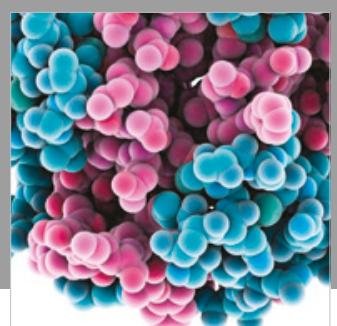

ournal of

Diabetes Research

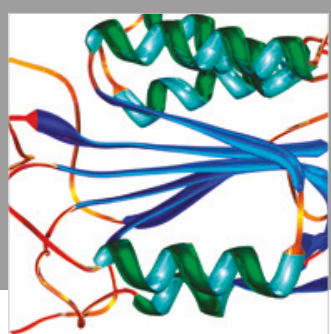

Disease Markers
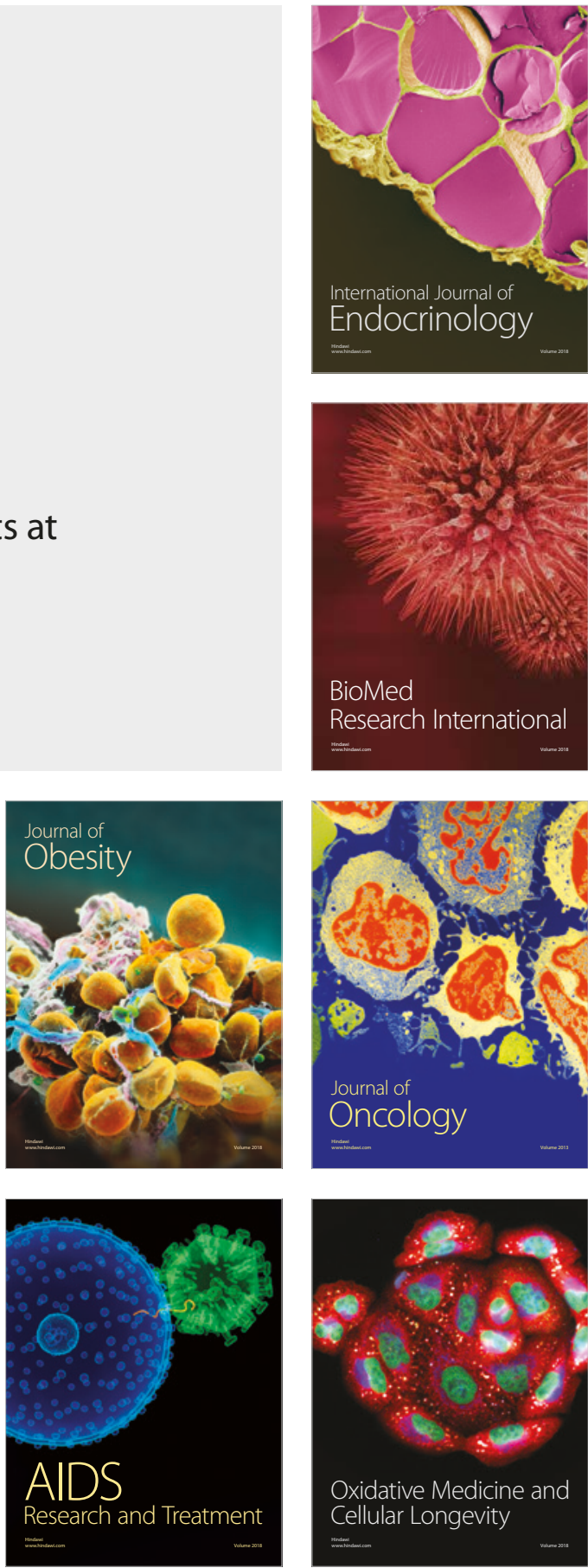